PROCEEDINGS OF THE

AMERICAN MATHEMATICAL SOCIETY

Volume 133, Number 2, Pages 493-499

S 0002-9939(04)07551-3

Article electronically published on August 20, 2004

\title{
THE CAYLEY TRANSFORM OF LINEAR RELATIONS
}

\author{
MERCEDES FERNANDEZ MIRANDA AND JEAN-PHILIPPE LABROUSSE
}

(Communicated by Joseph A. Ball)

\begin{abstract}
This paper extends the definition of the Cayley transform defined for symmetric linear operators to any linear relation.
\end{abstract}

\section{INTRODUCTION}

Let $\mathcal{H}$ be a complex Hilbert space with scalar product $(.,$.$) and norm \|.\| . \mathcal{H} \oplus \mathcal{H}$ will denote the direct sum of $\mathcal{H}$ with itself, with its natural Hilbert space structure. The notion of linear relation on a normed space, which was first introduced by $\mathrm{R}$. Arens in [1] (1961), generalizes the notion of linear operator (it is equivalent to the notion of multivalued operator and a general introduction can be found in [3]). The set of such linear relations on $\mathcal{H}$ will be denoted here by $\operatorname{Lr}(\mathcal{H})$ while $L R(\mathcal{H})$ will denote the set of closed elements of $\operatorname{Lr}(\mathcal{H})$ (i.e. those that have a closed graph in $\mathcal{H} \oplus \mathcal{H})$. The concept of inverse of an element and the operations of multiplication by a scalar, addition and composition can easily be generalized to linear relations.

The concepts of symmetric and selfadjoint operators readily extend to $L R(\mathcal{H})$, and the von Neuman theorem about selfadjoint extensions of symmetric operators can also be extended to $L R(\mathcal{H})$. This was done in [2] by making use of the Cayley transform of linear relations. In that paper, however, the definition of the Cayley transform was extended only to $L R(\mathcal{H})$, via the use of the orthogonal projection on the graph of a closed linear relation.

In this paper we want to show that the Cayley transform of a linear relation can be defined directly (as for operators) by an algebraic formula and can therefore be extended to $\operatorname{Lr}(\mathcal{H})$.

The Cayley transform $C(E)$ of an operator $E$ such that $E+i I$ has a trivial kernel is usually defined as follows:

$$
C(E)=(E-i I)(E+i I)^{-1}=I-2 i(E+i I)^{-1} .
$$

The two expressions in that definition coincide when $E$ is an operator, but this is not in general the case when $E$ is a linear relation as is shown below by Corollary 3.3 and it turns out that it is the second expression that yields the correct definition. In fact, here as in [2, we shall deal with a small variation of the Cayley transform - the $Z$ transform - which yields the additional "desirable" property of being an involution:

$$
Z(E)=-i C(E)=-i I-2(E+i I)^{-1}
$$

Received by the editors March 5, 2003 and, in revised form, October 15, 2003.

2000 Mathematics Subject Classification. Primary 47A05, 47A06.

Key words and phrases. Linear relation, operator, Cayley transform. 
In $\S 1$ we recall basic definitions and properties of linear relations which are needed for the sequel.

The main result of $\S 2$ is the extension (due to H. de Snoo) of Stone's formula for the orthogonal projection on the graph of a closed linear relation (a more complete presentation can be found in [4]).

Finally in $\S 3$ the Cayley transform of linear relations is defined and is shown to be equivalent to the one given in 2 for closed linear relations. Some of the results of [2] are then generalized to not necessarily closed linear relations.

\section{LINEAR RELATIONS}

Let $J$ and $K$ denote the symmetric unitary operators on $\mathcal{H} \oplus \mathcal{H}$ described by the matrices:

$$
J=\left(\begin{array}{cc}
I & 0 \\
0 & -I
\end{array}\right) ; \quad K=\left(\begin{array}{cc}
0 & -i I \\
i I & 0
\end{array}\right) \text {. }
$$

We shall also use $U=(J+K) / \sqrt{2}, P_{1}=(I+J) / 2$, and $P_{2}=(I-J) / 2$. Finally, let $\mathcal{H}_{1}=\mathcal{H} \oplus\{0\}=P_{1}(\mathcal{H} \oplus \mathcal{H})$ and $\mathcal{H}_{2}=\{0\} \oplus \mathcal{H}=P_{2}(\mathcal{H} \oplus \mathcal{H})$.

Proposition 1.1. $J K+K J=0$.

Proof. Obvious.

Definition 1.2. Let $\mathcal{P}(\mathcal{H})$ denote the set of all subsets of $\mathcal{H}$. Then $\mathcal{P}(\mathcal{H})$ can be given a linear space structure on $\mathbf{C}$ as follows: If $M, N \in \mathcal{P}(\mathcal{H})$, set $M+N=$ $\{z \mid z=m+n, m \in M, n \in N\}$ and $\forall \lambda \in \mathbf{C}$, set $\lambda M=\{\lambda z \mid z \in M\}$.

Definition 1.3. A linear relation $E$ in $\mathcal{H}$ is a mapping of a linear subspace $\tilde{D}(E)$ of $\mathcal{H}$ into $\mathcal{P}(\mathcal{H})$ such that

$$
\begin{gathered}
\forall x, y \in \tilde{D}(E), E(x+y)=E(x)+E(y), \\
\forall \lambda \in \mathbf{C}, \forall x \in \tilde{D}(E), E(\lambda x)=\lambda E(x) .
\end{gathered}
$$

Definition 1.4. Let $E$ be a linear relation in $\mathcal{H}$. We shall define the domain of $E$ by $D(E)=P_{1}(G(E))=\tilde{D}(E) \oplus\{0\}$, the range of $E$ by $R(E)=P_{2}(G(E))=$ $\{0\} \oplus \tilde{R}(E)$, and the kernel of $E$ by $N(E)=G(E) \cap \mathcal{H}_{1}=\tilde{N}(E) \oplus\{0\}$.

Then the graph of $E$ is the linear subspace of $\mathcal{H} \oplus \mathcal{H}$ given by $G(E)=\{(x, y) \mid x \in$ $\tilde{D}(E), y \in E(x)\}$.

Setting $G(E) \cap \mathcal{H}_{2}=\{0\} \oplus E(0)$ we shall call $E(0)$ the multivalued part of $E$. Note that if $E(0)=\{0\}$, then $E$ is an operator. The set of all bounded and everywhere defined linear operators on $\mathcal{H}$ will be denoted by $L(\mathcal{H})$.

When $E \in L R(\mathcal{H})$ we shall write $P_{E}$ for the orthogonal projection in $\mathcal{H} \oplus \mathcal{H}$ onto $G(E)$.

Example. Let $X, Y$ be two closed subspaces of $\mathcal{H}$ such that $X+Y=\mathcal{H}$. Then every $z \in \mathcal{H}$ can be written as $z=x+y$, with $x \in X$ and $y \in Y$.

Denote by $P$ the linear mapping of $\mathcal{H}$ into $\mathcal{P}(\mathcal{H})$ given by $P: z \mapsto\{x\}+X \cap Y$. Then $P \in L R(\mathcal{H})$.

It is easy to see that $\tilde{N}(P)=Y$ and $P(0)=X \cap Y$.

Definition 1.5. Let $E, F \in L R(\mathcal{H})$. Then

$$
g(E, F)=\left\|P_{E}-P_{F}\right\| \text {. }
$$

$L R(\mathcal{H})$ equipped with the metric $g$ (the so-called gap metric) is complete. 
Definition 1.6. Let $E \in \operatorname{Lr}(\mathcal{H})$ and $\lambda \in \mathbf{C}$. Then $\lambda E$ is the linear relation such that its graph is $G(\lambda E)=\{\{x, \lambda y\} \mid\{x, y\} \in G(E)\}$.

Definition 1.7. Let $E, F \in \operatorname{Lr}(\mathcal{H})$. Then $E+F$, the sum of $E$ and $F$, is the linear relation with graph $G=\{\{x, u\} \mid \exists y, \exists z$ such that $\{x, y\} \in G(E) ;\{x, z\} \in G(F)$ and $u=y+z\}$. Clearly $D(E+F)=D(E) \cap D(F)$ and $(E+F)(0)=E(0)+F(0)$.

Remark 1.8. The sum is associative and commutative. If 0 is the linear relation whose graph is $\mathcal{H}_{1}$, then clearly $\forall E \in \operatorname{Lr}(\mathcal{H}), 0+E=E+0=E$ so that 0 is a neutral element for the sum. Finally note that unless $E \in L(\mathcal{H}), E+(-E) \neq 0$ although $E+(-E)+E=E$ and $(-E)+E+(-E)=-E$ so that $-E$ is a generalized inverse of $E$ for the sum (for a survey of the theory of generalized inverses see [5]).

Definition 1.9. Let $E \in \operatorname{Lr}(\mathcal{H})$. Then the inverse $E^{-1}$ is the linear relation with graph $G\left(E^{-1}\right)=K J(G(E))=J K(G(E))$ (since $J K=-K J$ by Proposition 1.1). Clearly $\left(E^{-1}\right)^{-1}=E$.

Definition 1.10. Let $E, F \in \operatorname{Lr}(\mathcal{H})$. Then $F E$, the product of $E$ on the left by $F$, is the linear relation with graph $G=\{\{x, y\} \mid \exists z \in \mathcal{H}$ such that $\{x, z\} \in$ $G(E),\{z, y\} \in G(F)\}$.

Remark 1.11. The product is associative and noncommutative. If $I$ is the identity mapping on $\mathcal{H}$, then clearly $\forall E \in \operatorname{Lr}(\mathcal{H}), I E=E I=E$ so that $I$ is a neutral element for the product. Finally note that in general $E E^{-1} \neq I$ although $E E^{-1} E=$ $E$ and $E^{-1} E E^{-1}=E^{-1}$ so that $E^{-1}$ is a generalized inverse of $E$ for the product.

Definition 1.12. Let $E \in \operatorname{Lr}(\mathcal{H})$. Then the adjoint $E^{*}$ is the closed linear relation with graph $G\left(E^{*}\right)=K\left(G(E)^{\perp}\right)=(K G(E))^{\perp}$.

Proposition 1.13. Let $E \in \operatorname{Lr}(\mathcal{H})$. Then $\tilde{D}\left(E^{*}\right)=E(0)^{\perp}$.

Proof. Let $u \in \tilde{D}\left(E^{*}\right)$. Then $\exists v \in \mathcal{H}$ such that $\{u, v\} \in G\left(E^{*}\right)$. Moreover if $w \in$ $E(0)$, then $\{0, w\} \in G(E) \Leftrightarrow\{w, 0\} \perp G\left(E^{*}\right)$ so that: $(u, w)=(\{u, v\},\{w, 0\})=0$ so that $\tilde{D}\left(E^{*}\right) \subseteq E(0)^{\perp}$. Conversely if $u \perp D\left(E^{*}\right)$, then $\{u, 0\} \perp G\left(E^{*}\right)$, which implies that $\{0, u\} \in G(E) \Rightarrow u \in E(0)$ and the proposition follows.

Proposition 1.14. Let $E \in L R(\mathcal{H})$. Then $\left(E^{*}\right)^{-1}=\left(E^{-1}\right)^{*}$.

Proof. $G\left(\left(E^{*}\right)^{-1}\right)=J K\left(G\left(E^{*}\right)\right)=J K K\left(G(E)^{\perp}\right)=K\left([K J(G(E))]^{\perp}\right)=$ $G\left(\left(E^{-1}\right)^{*}\right)$.

Proposition 1.15. Let $E \in L R(\mathcal{H})$ and $F \in L(\mathcal{H})$. Then

$$
E^{*}+F^{*}=(E+F)^{*} .
$$

Proof. Let $x \in \tilde{D}\left(E^{*}\right)$, and let $\{x, m\} \in G\left(E^{*}\right)$. Then $\forall u \in \tilde{D}(E), \forall s$ with $\{u, s\} \in G(E),(\{u, s\},\{m,-x\})=0 \Leftrightarrow(u, m)=(s, x)$. Also, $\forall u, \forall x \in \mathcal{H}$, $\left(\{u, F u\},\left\{F^{*} x,-x\right\}\right)=0 \Leftrightarrow\left(u, F^{*} x\right)=(F u, x)$. Therefore $\forall u \in \tilde{D}(E) \forall s$, such that $\{u, s\} \in G(E) \Leftrightarrow\{u, s+F u\} \in G(E+F),\left(\{u, s+F u\},\left\{m+F^{*} x,-x\right\}\right)=$ $0 \Leftrightarrow\left(u, m+F^{*} x\right)=(s+F u, x)$ so that $\left\{x, m+F^{*} x\right\} \in G\left((E+F)^{*}\right)$ and consequently $G\left(E^{*}+F^{*}\right) \subseteq G\left((E+F)^{*}\right)$. Hence, since $E+F-F=E$, it follows that $G\left((E+F)^{*}-F^{*}\right) \subseteq G\left((E+F-F)^{*}\right)=G\left(E^{*}\right)$ so that $G\left((E+F)^{*}\right)=$ $G\left((E+F)^{*}-F^{*}+F^{*}\right) \subseteq G\left(E^{*}+F^{*}\right)$ Hence $(E+F)^{*}=E^{*}+F^{*}$. 


\section{The ORTHOGONAL PROJECTION ON THE GRAPH OF A CLOSED LINEAR RELATION}

Proposition 2.1 (Stone-de Snoo formula). Let $E \in L R(\mathcal{H})$, and let

$$
\left(\begin{array}{cc}
\Delta_{E} & \nabla_{E^{*}} \\
\nabla_{E} & I-\Delta_{E^{*}}
\end{array}\right)
$$

be the matrix representation of the orthogonal projection $P_{E}$ in $\mathcal{H} \oplus \mathcal{H}$ onto the graph of $E$. Then

(i) $\Delta_{E}=\left(I+E^{*} E\right)^{-1} \in L(\mathcal{H})$.

(ii) $\nabla_{E}=\left(I-P_{E(0)}\right) E \Delta_{E} \in L(\mathcal{H})$.

(iii) $\Delta_{E^{*}}=\left(I+E E^{*}\right)^{-1} \in L(\mathcal{H})$.

(iv) $\nabla_{E^{*}}=\left(I-P_{E^{*}(0)}\right) E^{*} \Delta_{E^{*}} \in L(\mathcal{H})$.

Proof. (i) Let $u \in \mathcal{H}$. Then by definition $\{u, 0\}=\left\{\Delta_{E} u, \nabla_{E} u\right\}+\{y,-x\}$ where $\{y,-x\} \in G(E)^{\perp} \Leftrightarrow\{x, y\} \in G\left(E^{*}\right)$. Hence $\nabla_{E} u=x \in \tilde{D}\left(E^{*}\right)=E(0)^{\perp}$ (Proposition 1.13) and $\left\{\Delta_{E} u, y\right\} \in G\left(E^{*} E\right) \Leftrightarrow\left\{\Delta_{E} u, y+\Delta_{E} u\right\}=\left\{\Delta_{E} u, u\right\} \in G\left(I+E^{*} E\right)$ so that finally $\left\{u, \Delta_{E} u\right\} \in G\left(\left(I+E^{*} E\right)^{-1}\right) \Rightarrow G\left(\Delta_{E}\right) \subseteq G\left(\left(I+E^{*} E\right)^{-1}\right)$. Now let $w \in \tilde{N}\left(I+E^{*} E\right)$. Then $\{w, 0\} \in G\left(I+E^{*} E\right) \Leftrightarrow\{w,-w\} \in G\left(E^{*} E\right)$ so that $\exists z \in \mathcal{H}$ such that $\{w, z\} \in G(E)$ and $\{z,-w\} \in G\left(E^{*}\right) \Leftrightarrow\{w, z\} \perp G(E)$ and therefore $\|w\|^{2}+\|z\|^{2}=0 \Rightarrow w=0$. Hence $\tilde{N}\left(I+E^{*} E\right)=\{0\}$ so that $\left(I+E^{*} E\right)^{-1}$ is an operator and consequently since $\tilde{D}(E)=\mathcal{H}, \Delta_{E}=\left(I+E^{*} E\right)^{-1}$. Since clearly $\Delta_{E}$ is closed, (i) is proved.

(ii) It is clear that $\left\{u, \nabla_{E} u\right\} \in G\left(E \Delta_{E}\right)$ and since $\nabla_{E} u \perp E(0)$ it follows that $G\left(\nabla_{E}\right) \subseteq G\left(\left(I-P_{E(0)}\right) E \Delta_{E}\right)$. As above, the fact that $\left(I-P_{E(0)}\right) E \Delta_{E}$ is an operator and $\tilde{D}\left(\left(I-P_{E(0)}\right) E \Delta_{E}\right)=\mathcal{H}$ implies that $\nabla_{E}=\left(I-P_{E(0)}\right) E \Delta_{E}$. Since clearly $\nabla_{E}$ is closed, (ii) is proved.

(iii) Let $v \in \mathcal{H}$. Then by definition $\{0, v\}=\left\{\nabla_{E^{*}} v, v-\Delta_{E^{*}} v\right\}+\{-t, s\}$ where $\{-t, s\} \in G(E)^{\perp} \Leftrightarrow\{s, t\} \in G\left(E^{*}\right)$. Hence $s=\Delta_{E *} v$ and $t=\nabla_{E *} v$. Also $\left\{\nabla_{E^{*}} v, v-\Delta_{E^{*}} v\right\} \in G(E) \Leftrightarrow\left\{v-\Delta_{E^{*}} v,-\nabla_{E^{*}} v\right\} \in G\left(E^{*}\right)^{\perp}$. Hence $\{v, 0\}=$ $\{s, t\}+\left\{v-\Delta_{E^{*}} v,-\nabla_{E^{*}} v\right\}$ so that (iii) and (iv) follow from (i) and (ii), exchanging $E$ with $E^{*}$.

Corollary 2.2. Let $E \in L R(\mathcal{H})$. Then $\nabla_{E}^{*}=\nabla_{E^{*}}$ and $\Delta_{E}$ and $\Delta_{E^{*}}$ are selfadjoint.

Proof. Immediate consequence of the fact that $P_{E}$ is orthogonal.

Corollary 2.3. Let $E \in L R(\mathcal{H})$. Then $\Delta_{E}^{2}+\nabla_{E^{*}} \nabla_{E}=\Delta_{E}, \Delta_{E} \nabla_{E^{*}}-\nabla_{E^{*}} \Delta_{E^{*}}=$ $0, \nabla_{E} \Delta_{E}-\Delta_{E^{*}} \nabla_{E}=0$, and $\nabla_{E} \nabla_{E^{*}}+\Delta_{E^{*}}^{2}=\Delta_{E^{*}}$.

Proof. Immediate consequence of the fact that $P_{E}$ is a projection.

Proposition 2.4. Let $E \in L R(\mathcal{H})$. Then

$$
\begin{aligned}
\forall u \in \mathcal{H},\left\|\Delta_{E} u\right\|^{2}+\left\|\nabla_{E} u\right\|^{2} \leq\left\|\Delta_{E} u\right\|\|u\| ; \\
\left\|\Delta_{E}\right\| \leq 1 ; \\
\left\|\nabla_{E}\right\| \leq \frac{1}{2} .
\end{aligned}
$$

Proof. Let $u \in \mathcal{H}$. Then $\{u, 0\}=\{s, t\}+\{x,-y\}$ with $\{s, t\} \in G(E)$ and $\{y, x\} \in$ $G\left(E^{*}\right)$ and $u=s+x, 0=t-y$. Then $t=y=\nabla_{E} u, s=\Delta_{E} u$ and $(s, u)-\|s\|^{2}-$ 
$\|t\|^{2}=(\{s, t\},\{x,-y\})=\left(P_{E}\{s, t\},\left(I-P_{E}\right)\{x,-y\}\right)=0$. Therefore,

$$
\|s\|^{2}+\|t\|^{2}=(s, u)
$$

so that $\|s\|^{2}+\|t\|^{2} \leq\|s\|\|u\|$, which establishes (2.2). Then $\forall u \in \mathcal{H},\left\|\Delta_{E} u\right\|^{2} \leq$ $\left\|\Delta_{E} u\right\|\|u\|$ so that $\forall u \in \mathcal{H},\left\|\Delta_{E} u\right\| \leq\|u\|$, which implies (2.3) and $\forall u \in \mathcal{H}$, $\left\|\Delta_{E} u\right\|^{2}+\left\|\nabla_{E} u\right\|^{2} \leq\left\|\Delta_{E} u\right\|^{2}+(1 / 4)\|u\|^{2}$ so that $\forall u \in \mathcal{H},\left\|\nabla_{E} u\right\|^{2} \leq(1 / 4)\|u\|^{2}$, which implies (2.4).

Corollary 2.5. Let $E \in L R(\mathcal{H})$. Then

(i) $\Delta_{E}$ is positive;

(ii) $\tilde{N}\left(\Delta_{E}\right)=E^{*}(0)=\tilde{D}(E)^{\perp}$.

Proof. (i) From Corollary 2.2 we know that $\Delta_{E}$ is selfadjoint and from (2.5) it follows that $\forall u \in \mathcal{H},\left(\Delta_{E} u, u\right) \geq\left\|\Delta_{E} u\right\|^{2} \geq 0$.

(ii) $u \in \tilde{N}\left(\Delta_{E}\right) \Leftrightarrow\{0, u\} \in G\left(I+E^{*} E\right) \Leftrightarrow\{0, u\} \in G\left(E^{*} E\right) \Rightarrow \exists z \in \mathcal{H}$ such that $\{0, z\} \in G(E)$ and $\{z, u\} \in G\left(E^{*}\right) \Rightarrow(\{z, 0\},\{z, u\})=0 \Rightarrow z=0$ so that $u \in E^{*}(0)$. Note that $E^{*}(0)=E^{*} E(0)$. Indeed it is clear that $E^{*}(0) \subseteq E^{*} E(0)$ and conversely if $v \in E^{*} E(0)$, then $\{0, v\} \in G\left(E^{*} E\right)$ so that $\left.\exists w \in \tilde{(} D\right)\left(E^{*}\right)$ such that $\{0, w\} \in G(E)$ and $\{w, v\} \in G\left(E^{*}\right)$. But then $\{w, 0\} \in G\left(E^{*}\right)^{\perp}$ so that $\|w\|^{2}=(\{w, 0\},\{w, v\})=0$ and consequently $\{0, v\}=\{w, v\} \in G\left(E^{*}\right)$ so that $v \in E^{*}(0)$. Hence $\{0, u\} \in G\left(E^{*}\right) \Leftrightarrow\{0, u\} \in G\left(E^{*} E\right) \Leftrightarrow\{0, u\} \in G\left(I+E^{*} E\right)$ $\Leftrightarrow u \in \tilde{N}\left(\Delta_{E}\right)$, and therefore the first equality is proved. The second equality follows from Proposition 1.13 .

\section{The extended Cayley transform}

Definition 3.1. Let $E$ be a linear relation. Then $Z(E)=-i I-2(E+i I)^{-1}$ is by definition the Cayley transform of $E$.

Proposition 3.2. Let $E \in \operatorname{Lr}(\mathcal{H})$ and let $F \in L(\mathcal{H})$. Then

$$
G\left[\left(E+F^{*}\right)(E+F)^{-1}\right]=G\left[I+\left(F^{*}-F\right)(E+F)^{-1}\right]+\{0\} \oplus E(0) .
$$

Proof. Let $\{u, v\} \in G\left[\left(E+F^{*}\right)(E+F)^{-1}\right]$. Then $\exists w \in \tilde{D}(E)$ such that $\{u, w\} \in$ $G\left[(E+F)^{-1}\right] \Leftrightarrow\{w, u\} \in G(E+F)$ and $\{w, v\} \in G\left(E+F^{*}\right)$. Then $\{w, u-F w\} \in$ $G(E)$ and $\left\{w, v-F^{*} w\right\} \in G(E)$ so that $\left\{0, F^{*} w-F w+u-v\right\} \in G(E)$. Also $\left\{u, F^{*} w-f\right\} \in G\left[\left(F^{*}-F\right)(E+F)^{-1}\right.$ so that $\{u, v\}=\left\{u,\left(F^{*}-F\right) w+u\right\}-$ $\left\{0,\left(F^{*}-F\right) w+u-v\right\} \in G\left[I+\left(F^{*}-F\right)(E+F)^{-1}\right]+\{0\} \oplus E(0)$. Conversely let $\{r, s\} \in G\left[I+\left(F^{*}-F\right)(E+F)^{-1}\right]+\{0\} \oplus E(0)$. Then $\exists x \in E(0)$ such that $\{u, v-u-x\} \in G\left[\left(F^{*}-F\right)(E+F)^{-1}\right]$ so that $\exists t \in \tilde{D}(E)$ such that $\{r, t\} \in$ $G\left[(E+F)^{-1}\right] \Leftrightarrow\{t, r\} \in G(E+F)$ and $s-r-x=\left(F^{*}-F\right) t$. Hence $\{t, r-F t\} \in$ $G(E) \Rightarrow\left\{t, s-F^{*} t\right\}=\{t, r-F t+x\} \in G(E)$, so that $\{t, s\} \in G\left(E+F^{*}\right)$ and consequently $\{r, s\} \in G\left[\left(E+F^{*}\right)(E+F)^{-1}\right]$.

Corollary 3.3. Let $E \in \operatorname{Lr}(\mathcal{H})$, and let $F \in L(\mathcal{H})$. Then if $E+F$ is injective and $E$ is not an operator,

$$
G\left[\left(E+F^{*}\right)(E+F)^{-1}\right] \neq G\left[I+\left(F^{*}-F\right)(E+F)^{-1}\right] .
$$

Proof. Under the hypothesis, $I+\left(F^{*}-F\right)(E+F)^{-1}$ is an operator (i.e. has a trivial multivalued part) while $E(0)$ is the nontrivial multivalued part of $\left(E+F^{*}\right)(E+F)^{-1}$. 
Proposition 3.4. Let $E \in L R(\mathcal{H})$ and $F=Z(E)$. Then $P_{F}=U P_{E} U$, which is how the Cayley transform of closed linear relations was defined in [2].

Proof. Let $u \in \mathcal{H}$. Then $\left\{\Delta_{E} u, \nabla_{E} u\right\} \in G(E)$ so that $\left\{-i \Delta_{E} u,-i \nabla_{E} u+\Delta_{E} u\right\} \in$ $G(E+i I)$. Hence

$$
-i \nabla_{E} u+\Delta_{E} u \in \tilde{R}(E+i I) .
$$

Similarly $\left\{\nabla_{E^{*}} u, u-\Delta_{E^{*}} u\right\} \in G(E)$, which implies that $\left\{\nabla_{E^{*}} u, u-\Delta_{E^{*}} u+\right.$ $\left.i \nabla_{E^{*}} u\right\} \in G(E+i I)$. Hence

$$
u-\Delta_{E^{*}} u+i \nabla_{E^{*}} u \in \tilde{R}(E+i I) .
$$

Then $v=(1 / 2)\left(-i \nabla_{E} u+\Delta_{E} u+u-\Delta_{E^{*}} u+i \nabla_{E^{*}} u\right) \in \tilde{R}(E+i I)=\tilde{D}(F)$ so that $\exists w \in \mathcal{H}$ such that $\{v, w\} \in G(F)$. Then $w=-i v+r$ with $\{v, r\} \in$ $G\left(-2(E+i I)^{-1}\right)$ or equivalently $\{-2 v, r\} \in G\left((E+i I)^{-1}\right) \Leftrightarrow\{r,-2 v\} \in G(E+i I)$. Then $\{r,-2 v\}+\left\{-i \Delta_{E} u,-i \nabla_{E} u+\Delta_{E} u\right\}+\left\{\nabla_{E^{*}} u, u-\Delta_{E^{*}} u+i \nabla_{E^{*}} u\right\}=\{r-$ $\left.i \Delta_{E} u+\nabla_{E^{*}} u, 0\right\} \in G(E+i I)$. Set $s=r-i \Delta_{E} u+\nabla_{E^{*}} u \in \tilde{N}(E+i I)=F(0)$. Hence $w=s+(1 / 2)\left(2 i \Delta_{E} u-2 \nabla_{E^{*}} u-i \Delta_{E} u+\nabla_{E^{*}} u-\nabla_{E} u-i u+i \Delta_{E^{*}} u\right)=$ $s+(1 / 2)\left(i \Delta_{E} u-\nabla_{E^{*}} u-\nabla_{E} u-i u+i \Delta_{E^{*}} u\right)$ and $\{v, w-s\} \in G(F)$. Now $\left\{\Delta_{E^{*}} u, \nabla_{E^{*}} u\right\} \in G\left(E^{*}\right)$ so that $\left\{\Delta_{E^{*}} u, \nabla_{E^{*}} u-i \Delta_{E^{*}} u\right\} \in G\left(E^{*}-i I\right)$ and therefore

$$
-\nabla_{E^{*}} u+i \Delta_{E^{*}} u \in \tilde{R}\left(E^{*}-i I\right) .
$$

Similarly $\left\{\nabla_{E} u, u-\Delta_{E} u\right\} \in G\left(E^{*}\right)$ so that $\left\{\nabla_{E} u, u-\Delta_{E} u-i \nabla_{E} u\right\} \in G\left(E^{*}-i I\right)$ and hence

$$
-i u+i \Delta_{E} u-\nabla_{E} u \in \tilde{R}\left(E^{*}-i I\right) .
$$

Using Propositions (1.14) and (1.15) it is easy to see that $F^{*}=i I-2\left(E^{*}-i I\right)^{-1}$. Then $w-s=-\nabla_{E^{*}} u+i \Delta_{E^{*}} u-i u+i \Delta_{E} u-\nabla_{E} u \in \tilde{R}\left(E^{*}-i I\right) \in \tilde{D}\left(F^{*}\right)$, so that $\exists x \in \mathcal{H}$ such that $\{w-s, x\} \in G\left(F^{*}\right)$. Then $x=i(w-s)+t$ with $\{w-s, t\} \in G\left(-2\left(E^{*}-i I\right)^{-1}\right)$ or equivalently $\{-2(w-s), t\} \in G\left(\left(E^{*}-i I\right)^{-1}\right) \Leftrightarrow$ $\{t,-2(w-s)\} \in G\left(E^{*}-i I\right)$. So $\{t,-2(w-s)\}-\left\{\Delta_{E^{*}} u, \nabla_{E^{*}} u-i \Delta_{E^{*}} u\right\}+$ $\left\{-i \nabla_{E} u,-i u+i \Delta_{E} u-\nabla_{E} u\right\}=\left\{t-\Delta_{E^{*}} u-i \nabla_{E} u, 0\right\} \in G\left(E^{*}-i I\right)$. Set $y=$ $t-\Delta_{E^{*}} u-i \nabla_{E} u \in \tilde{N}\left(E^{*}-i I\right)=F^{*}(0)$. Then

$$
\begin{aligned}
x & =y+(1 / 2)\left(2 \Delta_{E^{*}} u+2 i \nabla_{E} u-\Delta_{E} u-i \nabla_{E^{*}} u-i \nabla_{E} u+u-\Delta_{E^{*}} u\right) \\
& =y+(1 / 2)\left(\Delta_{E^{*}} u+i \nabla_{E} u-\Delta_{E} u-i \nabla_{E^{*}} u+u\right)=y+u-v .
\end{aligned}
$$

Since $\{v, x\} \in G\left(F^{*} F\right)$, it follows that $\{v, x+v\}=\{v, u+y\} \in G\left(I+F^{*} F\right)$, which shows that $\{v, u\} \in G\left(I+F^{*} F\right) \Leftrightarrow v=\Delta_{F} u$ so that

$$
\Delta_{F}=(1 / 2)\left(\Delta_{E}+i \nabla_{E^{*}}-i \nabla_{E}+I-\Delta_{E^{*}}\right) .
$$

Quite analogously it can be shown that

$$
\Delta_{F^{*}}=(1 / 2)\left(\Delta_{E^{*}}-i \nabla_{E}+i \nabla_{E^{*}}+I-\Delta_{E}\right) .
$$

Finally let $\{u, z\} \in G\left(F \Delta_{F}\right)$. Then $\exists v$ such that $v=\Delta_{F} u$ and $\{v, z\} \in G(F)$. As above, it follows that $z=-i v+m$ with $\{v, m\} \in G\left(-2(E+i I)^{-1}\right)$, which is equivalent to $\{m,-2 v\} \in G(E+i I)$. Hence, using (3.1) and (3.2), we get $\{n, 0\}=\left\{m-i \nabla_{E} u+\nabla_{E^{*}} u, 0\right\} \in G(E+i I)$ so that $\{0, n\} \in F(0)=F \Delta_{F}(0)$ and consequently $\{u, z-n\} \in G\left(F \Delta_{F}\right)$. Therefore, since $z=-i v+m=$ $(1 / 2)\left(i \Delta_{E}-\nabla_{E^{*}}-\nabla_{E}-i I+i \Delta_{E^{*}}\right) u+n$ and using (3.3) and (3.4) we see that $z-n \in \tilde{R}\left(E^{*}-i I\right)=\tilde{D}\left(F^{*}\right)=F(0)^{\perp}$. It follows that $z-n=\nabla_{F} u$. Hence $\nabla_{F}=(1 / 2)\left(i \Delta_{E}-\nabla_{E^{*}}-\nabla_{E}-i I+i \Delta_{E^{*}}\right)$ and taking adjoints $\nabla_{F^{*}}=\left(\nabla_{F}\right)^{*}=$ 
$(1 / 2)\left(-i \Delta_{E}-\nabla_{E}-\nabla_{E^{*}}+i I-i \Delta_{E^{*}}\right)$. To establish the proposition it now suffices to observe that the coefficients in the matrices representing $P_{F}$ and $U P_{E} U$ are the same.

Corollary 3.5. The transform $Z$ is an isometry on $L R(\mathcal{H})$, i.e. $\forall E, F \in L R(\mathcal{H})$, $g(Z(E), Z(F))=g(E, F)$.

Proof. $g(Z(E), Z(F))=\left\|P_{Z(E)}-P_{Z(F)}\right\|=\left\|U\left(P_{E}-P_{F}\right) U\right\|=\left\|P_{E}-P_{F}\right\|=$ $g(E, F)$.

Proposition 3.6. Let $E$ be a linear relation (not necessarily closed). Then $Z(Z(E)$ ) $=E$. This property is the reason for our modification (by a factor $-i$ ) of the usual definition of the Cayley transform.

Proof. Let $F=Z(E)$. Then

$$
\begin{aligned}
F+i I=-2(E+i I)^{-1} & \Leftrightarrow(F+i I)^{-1}=-(1 / 2)(E+i I) \\
& \Leftrightarrow E+i I=-2(F+i I)^{-1} \Leftrightarrow E=Z(F)
\end{aligned}
$$

so that $E=Z(Z(E))$.

Proposition 3.7. Let $E$ be a linear relation (not necessarily closed). Then $Z\left(E^{-1}\right)$ $=(Z(E))^{-1}$.

Proof. Let $F=Z(E)$. Then we have $(u, v) \in G(F) \Leftrightarrow v=-i u+r$ and $\{u, r\} \in$ $G\left(-2(E+i I)^{-1}\right) \Leftrightarrow v=-i u+r$ and $\{-2 u, r\} \in G\left((E+i I)^{-1}\right) \Leftrightarrow v=-i u+r$ and $\{r,-2 u\} \in G(E+i I) \Leftrightarrow v=-i u+r$ and $\{r,-2 u-i r\} \in G(E) \Leftrightarrow v=-i u+r$ and $\{-2 u-i r, r\} \in G\left(E^{-1}\right) \Leftrightarrow v=-i u+r$ and $\{-2 u-i r, 2 r-2 i u\} \in G\left(E^{-1}+i I\right) \Leftrightarrow v=$ $-i u+r$ and $\{2 v,-2 u-i r\} \in G\left(\left(E^{-1}+i I\right)^{-1}\right) \Leftrightarrow u=2 u+i r-i v$ and $\{v, 2 u+i r\} \in$ $G\left(-2\left(E^{-1}+i I\right)^{-1}\right) \Leftrightarrow\{v, u\}=\{v, 2 u+i r-i v\} \in G\left(Z\left(E^{-1}\right)\right)$ so that $\{v, u\} \in$ $G\left((Z(E))^{-1}\right) \Leftrightarrow\{v, u\} \in G\left(Z\left(E^{-1}\right)\right)$, which establishes the proposition.

\section{REFERENCES}

[1] R. ARENS, Operational calculus of linear relations, Pacific J. Math. 11, (1961), pp. 9-23. MR 23:A517

[2] Z. BOULMAAROUF and J-Ph. LABROUSSE, The Cayley transform of linear relations, J. Egyptian Math. Soc. 2, (1994), pp. 53-65. MR 96a:47034

[3] R. CROSS, Multivalued linear operators, Marcel Dekker, Inc., New York (1998). MR 99j:47003

[4] Y. MEZROUI, Projection orthogonale sur le graphe d'une relation linéaire fermée, Trans. Amer. Math. Soc. 352(6), (2000), pp. 2789-2800. MR 2000j:47003

[5] M. Z. NASHED (ed.), General inverses and applications, Academic Press, New York (1976). MR 56:9943

Departamento de Matemáticas, Universidad de Antofagasta, Angamos 601, AntofaGasta, Chile

E-mail address: mfernandez@uantof.cl

Université de Nice-Sophia Antipolis, laboratoire J. A. Dieudonné, UMR du CNRS Nô 6621, Parc Valrose, Nice Cedex 02, France

E-mail address: labro@math.unice.fr 